\title{
Pour une analyse énonciative engagée et responsable des discours médiatiques
}

Entretien avec Alain Rabatel par Michèle Monte

\section{Alain Rabatel et Michèle Monte}

\section{OpenEdition}

\section{Journals}

\section{Édition électronique}

URL : https://journals.openedition.org/mots/22693

DOI : $10.4000 /$ mots. 22693

ISBN : 978-2-84788-893-5

ISSN : 1960-6001

\section{Éditeur}

ENS Éditions

\section{Édition imprimée}

Date de publication : 9 mars 2017

Pagination : 117-132

ISBN : $978-2-84788-892-8$

ISSN : 0243-6450

\section{Référence électronique}

Alain Rabatel et Michèle Monte, "Pour une analyse énonciative engagée et responsable des discours médiatiques », Mots. Les langages du politique [En ligne], 113 | 2017, mis en ligne le 09 mars 2017. consulté le 22 avril 2022. URL : http://journals.openedition.org/mots/22693 ; DOI : https://doi.org/ $10.4000 /$ mots. 22693 


\section{« Pour une analyse énonciative engagée et responsable des discours médiatiques $"{ }^{*}$}

MOTS. Les langages du politique : Vos travaux sur l'énonciation et le point de vue sont une référence pour de nombreux linguistes, mais ceux que vous avez consacrés plus particulièrement aux discours médiatiques - par exemple sur le traitement des suicides à France Télécom ou du début du septennat de Nicolas Sarkozy - sont moins connus. Pouvez-vous nous dire à partir de quelles problématiques vous avez abordé ces corpus?

Alain RABATEL : Une première réponse sera de dire que ces problématiques linguistiques sont, au plan théorique et méthodologique, les mêmes que celles que j'ai mises à contribution pour les textes littéraires, à savoir un ancrage énonciatif radical, qui analyse la construction des objets du discours (leur «référenciation », j’y reviendrai) et y lit, à partir d'elle, le(s) point(s) de vue des énonciateurs sous-jacents, sans compter, bien évidemment, les cas où les points de vue se disent à travers des opinions, des jugements de valeurs explicites. Cette distinction renvoie aux deux dimensions complémentaires de l'argumentation, l'argumentation directe, qui passe par des conduites argumentatives explicites, des arguments, des connecteurs, mais aussi l'argumentation indirecte qui repose sur les inférences qu'on tire de la construction des objets du discours, très efficace parce qu'elle argumente sans y paraître. De tels phénomènes sont légion dans les fictions, mais ils jouent un rôle fondamental dans les textes médiatiques, notamment ceux qui affichent une prétention à l'objectivité en se retranchant derrière une visée informative. J'essaie également de rendre compte des phénomènes graduels d'accord, de désaccord, à travers les notions de prise en charge et de postures énonciatives. J'accorde aussi une

* Alain Rabatel est professeur à l'École supérieure du professorat et de l’Éducation (ÉSPÉ) de l'académie de Lyon (Université Claude-Bernard Lyon 1) et membre du laboratoire Interactions, corpus, apprentissages et interactions (ICAR, UMR CNRS nº 5191). Il travaille principalement sur la prise en charge énonciative, l'effet point de vue, et les formes indirectes d'argumentation en lien avec l'effacement énonciatif. Son analyse de l'énonciation intègre la dimension interactionnelle des discours, qu'ils soient oraux ou écrits, monogérés ou polygérés. Dans un ouvrage collectif récent (Rabatel, 2015a), il revient sur son parcours en énonciation en insistant sur ses analyses des textes narratifs. Dans l'entretien qu'il a bien voulu accorder à la revue Mots. Les langages du politique et qui est retranscrit ci-dessous, nous nous sommes au contraire centrés sur les points de contact entre ses travaux et l'analyse des discours politiques. Entretien réalisé par Michèle Monte au printemps 2016. 
grande importance aux questions interprétatives : les interactions médiatiques reposent toujours sur des calculs interprétatifs antérieurs ou concomitants aux positionnements des locuteurs/interactants. Et ces positionnements n'ayant pas qu'une dimension épistémique (certes fondamentale), il faut aussi prendre en compte la question des valeurs, des affects, des émotions, des axiologies des locuteurs/énonciateurs. Enfin, un dernier prisme à travers lequel j'ai choisi et investigué mes corpus médiatiques cherche toujours à concilier l'analyse linguistique avec des questions sociales (à fortes retombées politiques et épistémologiques), ainsi qu'avec les problématiques de la responsabilité et de l'engagement, qui valent pour le chercheur comme pour les auteurs des discours.

MOTS. Les langages du politique : Quels sont les résultats les plus marquants de vos analyses?

Alain RABATEL : Je précise tout d'abord que mon travail porte essentiellement sur les médias écrits. Un résultat significatif concerne l'expression indirecte des points de vue : le locuteur simule un effacement d'où découle une impression d'objectivité, comme si le discours des faits était objectif et que seul le commentaire était subjectif. Mais même en l'absence de commentaire explicite, la construction de la réalité référentielle pèse sur la lecture des évènements, ne serait-ce qu'à travers la catégorisation. J'ai ainsi montré comment des formes d'écriture (à la mode), par exemple l'habitude d'écrire des textes de plus en plus courts, avec un simple montage de citations convergentes ou divergentes, ou de «faits bruts », apparemment sans intervention directe du journaliste, permettent, uniquement via la sélection ${ }^{1}$, de gommer des pans entiers de la réalité, d'acteurs, de forces, d'évènements, de formes de pensée et de vie. Ainsi de la façon dont s'écrit, juste avant le vote, la campagne électorale concernant le référendum sur le traité constitutionnel de Maastricht, qui réduit les opposants au traité à un rôle sporadique, alors qu'ils ont structuré le débat, et les présente uniquement à travers le prisme de citations de leurs adversaires qui les accusent de populisme (Rabatel, 2006). J'ai prolongé ce travail sur le discours primaire pris en charge par les journalistes ${ }^{2}$ en étudiant comment leurs questions, lors des primaires socialistes de 2006 , orientent les débats dans des directions politiciennes ou des conflits de surface, ignorent d'autres questionnements possibles relatifs aux classes défavorisées, entrainant du même coup une invisibilité médiatique et politique de ces couches sociales et des problématiques politiques qu'elles soulèvent (Rabatel, 2008a). La couverture médiatique des suicides à France Télécom (Rabatel, 2010c, 2011a) offre également matière à réflexion sur les responsabilités des journalistes dans ces phénomènes d’invisibilité.

1. Dont on ne se justifie pas, en l'absence de discours primaire...

2. À la différence de rubriques comme «Désintox» ou "Les Décodeurs », qui pratiquent la critique des sources et n'hésitent pas à discuter la pertinence des analyses des leaders politiques et font donc plus qu'en «venir aux faits», puisqu'ils en discutent la construction (Rabatel, 2014a). 
La responsabilité (Rabatel et Chauvin, 2006 ; Rabatel et Koren, 2008), plus large que la prise en charge énonciative, est une notion linguistique, contrairement à ce qu'on prétend, car elle interroge les journalistes sur leur choix de planification et de mise en discours (Rabatel, 2006, 2017a); elle est également distincte de la déontologie, qui mérite par ailleurs d’être interrogée, car on peut en respecter les règles et malgré tout être interpellé à l'aune d'un questionnement sur la responsabilité éthique. J'ai aussi posé ces questions de responsabilité journalistique dans différents genres, e. $g$. à travers la façon de rapporter les propos des femmes et des hommes politiques (Rabatel, 2014b), certaines formes de spectacularisation complaisantes à peine contrebalancées par une distanciation ironique ou cynique à propos de portraits de N. Sarkozy (Rabatel, 2010a), ou les roueries du jeu avec les présupposés et les sous-entendus lors de la révélation de la liaison de F. Hollande avec J. Gayet (Rabatel, 2017b). J'ajoute que, si la plupart de mes travaux sur les médias pointent sur des formes du contenu ou de l'expression discutables, d'autres me donnent l'occasion d'analyser des pratiques positives 3 ou innovantes, par exemple le rôle critique et ironique des discours représentés dans des dessins (Rabatel, 2004) ou dans des titres qui jouent avec des noms propres et la figure de l'à-peu-près (Rabatel, 2011a), celui des citations complexes et des hyperliens des sites sur le World Wide Web, tel Arrêt sur images (Rabatel, 2010b), celui du "fact-checking", même si la formule mérite d’être questionnée sur certaines de ses limites, notamment le fait de n'interroger que les propos des dirigeants politiques, et jamais ceux du monde de la finance ou des entreprises, sans doute parce que la presse, soi-disant indépendante... ne l'est pas autant que les journalistes aiment à le dire, du moins à l'égard des puissance d'argent (Rabatel, 2013a).

MOTS. Les langages du politique : En quoi la notion de point de vue vous paraitelle éclairante pour l'analyse de discours?

Alain RABATEL : La notion de point de vue est une notion complexe, dont on peut prendre la mesure d'abord au plan des emplois ordinaires, non scientifiques. Un point de vue, c'est un lieu surplombant qui offre une belle vue, ayant à voir avec la notion d'ordre. Le plus souvent, cet ordre provient de la façon dont la personne dispose ou représente les objets. Ainsi font le peintre, calculant la taille des objets selon leur position relative par rapport à un centre de perspective orientant un point de fuite, ou le romancier décrivant un personnage ou racontant un évènement sous un jour favorable ou défavorable (Rabatel, 2009a). Ces exemples rappellent que le point de vue envisage des données plus ou moins complexes en fonction d'une perspective humaine qui

3. Du point de vue foncièrement éthico-politique qui est le mien, qui considère que tout doit être tenté pour faire société. C'est moins un point de vue normatif éthique abstrait qu'un point de vue tout pragmatique qui constate les dégâts causés par les libertés prises avec cette ambition politique (Rabatel, 2017a). 
hiérarchise, en intriquant dimensions perceptives et cognitives, associées à des données évaluatives, émotives, visant souvent des fins esthétiques et hédonistes.

Les dimensions cognitives, évaluatives et émotives se retrouvent dans l'usage courant du terme - même si cet emploi minore la dimension perceptive : «avoir un point de vue », c'est avoir une opinion. Mais l'opinion n'est pas un jugement, c'est plutôt un savoir hérité et/ou qui procède de l'expérience (personnelle ou collective), sans prendre la forme distanciée, rationalisée du jugement critique, qui, lui, transforme l'opinion, partielle et partiale, en jugement plus global, acceptable par tous : d'où les expressions «c'est ton point de vue ", "c'est un point de vue comme un autre », qui soulignent toutes la subjectivité et l’incomplétude du point de vue.

Toutefois la signification de la notion de point de vue ne se réduit pas à des idées reçues. Une simple requête sur l'internet montrerait qu'il existe à foison des expressions du type : "d'un point de vue politique», "d'un point de vue économique», «d'un point de vue philosophique », "d'un point de vue cartésien », etc. Toutes ces formulations pourraient être remplacées par l'expression «cadre (notionnel)» ou «paradigme (théorique)». Ici, la notion de point de vue souligne que la perspective est intellectualisée, dépend de principes qui pèsent sur la définition de l'objet comme sur son analyse.

Cette complexité des usages ordinaires du mot en fait un bon candidat à un usage technique, scientifique, dans les sciences du langage : il devient alors un concept pour lequel je recours aux abréviations majuscules PDV 4 : un PDV dénote et prédique quelque chose à propos d'un objet, alors que le choix des mots, de leur ordre, témoigne du point de vue de l'énonciateur sur ce même objet5. L'approche pragma-énonciative du point de vue est ainsi un levier pour prendre en compte l'opacité fondamentale des objets discursifs, dès les opérations de «référenciation » et de prédication.

Le PDV est une problématique plus complexe que celle de la voix et du discours rapporté, car si voix et discours rapportés renvoient bien à des PDV exprimés plus ou moins directement par leur auteur, il devient plus difficile de repérer le PDV d'un tel, dès lors que sa voix ne se fait plus entendre : cette diffi-

4. J'utilise avec distance l'opposition notion (représentation triviale, a- ou préscientifique des objets sociaux en usage) versus concept (théorisation savante de l'Objet de recherche). Dans les années soixante-dix du siècle précédent, dans la mouvance marxiste dont je me réclamais, seul le concept méritait attention et son maniement permettait de distinguer les professionnels scientifiques de la politique des amateurs... La distinction reste pertinente (en ce qu'elle rend compte des efforts de la théorie), mais il faut se méfier des illusions d'une «coupure radicale» (Althusser) entre Théorie et pratiques analytiques «illusoires/aliénées», "sauvages». C'est pourquoi j'utilise indifféremment les expressions la «notion de PDV» ou « le concept de PDV» et, plus souvent, l'expression plus englobante de problématique (générale) du PDV, articulant les théorisations savantes et les usages populaires de l'expression, qui s'éclairent partiellement.

5. Voir Rabatel 2012a, p. 15-20 pour des exemplifications à partir de textes littéraires et médiatiques. 
culté, caractéristique du discours indirect libre, qui n'est pas vraiment un « discours", est encore plus nette chaque fois qu'un énoncé - de surcroît en troisième personne ${ }^{6}$, comme souvent le discours indirect libre! - décrit, informe, raconte, en laissant penser que, puisqu'il n'y a pas d'opinion explicite, il n'y aurait pas de PDV et que le discours serait objectif. Bref, analyser des PDV quand il semble (naïvement) ne pas y avoir, voilà qui est précieux pour l'étude des médias! Une autre dimension positive est de rendre compte du caractère souvent co-construit des PDV (qu'ils soient singuliers, collectifs, anonymes, doxaux ou non). Cette dimension, particulièrement mise en relief à travers les postures énonciatives (j’y reviendrai plus loin), aide à problématiser des énoncés qui pourraient paraître plus plats et transparents qu'ils ne sont : là encore, quel filon pour l'analyse linguistique et critique des discours médiatiques!

MOTS. Les langages du politique : Pouvez-vous préciser ce qu'est la «référenciation »?

Alain RABATEL : Le référent, c'est le réel extérieur à la langue; quant à la référenciation, c'est une opération linguistique qui concerne le choix des expressions référentielles, ou, plus largement, la construction des univers du discours, indépendamment de la question des rapports entre ces univers et la réalité extralinguistique. Cette opération relève de part en part de choix énonciatifs, donc des points de vue que les énonciateurs ont sur les objets. On ne peut en effet réduire l'énonciation au je/ici/maintenant et il faut être conscient que l'énonciateur est partout, pas seulement dans les marques indexicales. Mais j'articule le plus souvent la référenciation avec l'énonciation (au point que c'est pour moi la même chose, comme le recto et le verso d'une feuille de papier), pour rappeler aussi que la « référenciation» ne s'entend pas en un sens platement et «objectivistement référentialiste» et permet d'influer sur les interprétations comme sur les actions. Ainsi, dans Rabatel 2013b, la façon dont l'avocat général (re)construit empathiquement le passage à l'homicide de la part de la femme victime de violences conjugales, en la nommant en tant que «mère de ses enfants», en faisant d'elle le symbole de «ces femmes que personne n'écoute », à commencer par les institutions qui devraient les aider, permet de comprendre que c'est le refus d'exposer ses enfants aux mêmes mauvais traitements que ceux qu'elle a subis qui la conduit au meurtre, tout comme sa solitude. La «référenciation » de ce meurtre par amour maternel et par non assistance à personne en danger permet d'entrer dans les raisons de cette femme, mais aussi dans celles de l'avocat général, et le lecteur comprend pourquoi ce dernier appellera pour la première fois dans ce genre d'affaires à un non lieu qui sera voté par les jurés.

6. Comme si la subjectivité ne passait que par la première personne - et comme si tout énoncé en je était forcément et fortement subjectivant... 
MOTS. Les langages du politique : Vous avez défini trois types de postures énonciatives dans les interactions dialogiques - qu'elles se fassent en contexte dialogal ou dans des textes monogérés : en quoi ces catégories peuvent-elles nous aider à penser l'argumentation et le conflit socio-discursif?

Alain RABATEL : En effet, ces postures énonciatives permettent de complexifier la notion de consensus, souvent mise en avant dans les théories interactionnelles, en appui sur le principe coopératif de Grice, avec l’idée qu'on ne peut interagir qu'en étant fortement coopératif. De fait, il faut coopérer, mais cela ne signifie pas que le consensus soit une notion homogène. II arrive que les deux sources de PDV prennent en charge le PDV co-construit, qu'elles font leur ; la co-énonciation, c'est l'accord parfait, si je puis dire, une sorte de quasi fusion des voix, un «nous » énonciatif qui se propage à tous les éléments de la matérialité discursive. Mais il est une deuxième forme d'accord, apparent, que je nomme la sur-énonciation, qui s'éloigne déjà de l'accord, malgré les apparences : dans ce cas, un des deux énonciateurs, tout en affirmant être d'accord avec le PDV de l'autre, le reformule d'une façon qui fait entendre une fêlure, et qui correspond à une sorte de coup de force discret par lequel, derrière l'accord, le même PDV est orienté dans un sens un peu différent $e . g$. avec un cadre de validité plus restreint ou plus large, ou une orientation argumentative différente. C'est là une façon pour le sur-énonciateur de marquer sa position surplombante par rapport à l'autre, en espérant que la nouvelle formulation du PDV oriente les échanges dans un sens qui n'était pas celui qu'on pouvait imaginer après sa première formulation. Un pas plus loin vers le dissensus, c'est la sous-énonciation, qui consiste en le fait de reprendre le PDV de l'autre, mais sous une forme distanciée7. Ici, le PDV de l'autre n'est plus pris en charge, il est seulement pris en compte (Rabatel, 2009b, 2012a). On voit ainsi que l'accord est un ensemble graduel, fragile, labile, avec des marques d'accord très diverses dans leur nature comme dans leurs effets - accord qui se donne linguistiquement comme effectif ${ }^{8}$, accord apparent à son profit exclusif, accord apparent distancié, avec dégagement énonciatif discret.

J'ai surtout analysé le fonctionnement des postures dans la confrontation des PDV à travers des reprises, reformulations, à l'échelle des énoncés, ou de plusieurs énoncés rapprochés. Mais on ne peut se limiter aux phénomènes contigus : il existe des reformulations ou modulations à distance (dans le texte ou l'interdiscours) de telle ou telle formule (Krieg-Planque, 2009) comme le montre le formidable travail de M.-F. Florea (2015) sur les nécrologies et sur les postures

7. On trouvera des exemplifications de ces postures notamment dans Rabatel, 2005, 2012b, 2015 b.

8. Car c'est autre chose que la question de savoir si cet accord est sincère, réel dans la tête des individus : ce qui m'intéresse, c'est la façon dont les choses se disent, et, partant, dont elles influent sur les interprétations des tiers (notamment des lecteurs) et aussi sur les interprétations des allocutaires dans le dialogue ou des co-énonciateurs, dans les écrits monogérés. 
énonciatives de sous-énonciation dans le blâme (car il arrive que les nécrologies abordent ponctuellement des aspects négatifs de la vie des défunts, ne serait-ce que sous la forme de discours rapportés), et, plus largement, la posture de surénonciation dans l'éloge, qui correspond à la visée épidictique fondamentale de ce genre de discours. Les postures sont un outil précieux pour l'analyse du positionnement de soi et l'étude linguistique des idéologies, des formations discursives. Elles pourraient aider à typologiser certaines pratiques médiatiques : Qui sur-énonce, sous-énonce, co-énonce? Quand (au début d'une interaction, dans des phases reformulatives, initiatives, conclusives, des relances)? Où (c'est-à-dire dans quel type de journal, de situation médiatique, de rubrique, au début, à la fin, à la charnière des paragraphes, etc.) ? Comment (par le lexique, la syntaxe, des procédés rhétoriques, la prosodie, le graphisme, la ponctuation - noire, blanche, grise - la planification, la mise en page, etc.) ? Pourquoi? Etc. Les médias sont une mine pour traiter ces questions, par exemple avec le rôle énonciatif de la titraille par rapport au contenu des articles, celui du sous-titrage des photos, de la mise en page, de l'organisation du discours, des clausules distanciées dans la sur-énonciation (Rabatel 2017b), qui rejoint le rôle de la rédaction par rapport à celui des journalistes de la rédaction et plus encore par rapport aux journalistes des agences ou aux journalistes free-lance. De même, la connivence, le persiflage (Rabatel 2010a, 2015c), l'implicite construisent souvent une co-énonciation, tandis que les reformulations distanciées, mais sans critique directe du journaliste, alimentent une posture discrète de sous-énonciation.

MOTS. Les langages du politique : Dans vos travaux récents sur les émotions, vous montrez comment l'empathie se manifeste linguistiquement et comment certains choix linguistiques sont susceptibles de nous permettre d'entrer dans la compréhension des sentiments et émotions d'autrui. En quoi ces travaux vous paraissent-ils importants pour l'analyse de discours et au-delà pour la construction de la citoyenneté?

Alain RABATEL : L'empathie est l'aptitude à se mettre à la place des autres (Berthoz, 2004). Si on veut l'analyser d'un point de vue linguistique, cela consiste à imaginer ce qu'ils peuvent percevoir, ressentir, penser, dire, faire, et donc à étudier l'expression des perceptions, des émotions, des pensées, des paroles, des actions des autres. Cela, c'est une forme d'empathie imaginative, projective, secondarisée, plus sophistiquée que les formes primaires d'empathie (voir les neurones miroirs, chez Rizzolatti et Sinigaglia, 2007) ou de sympathie9.

Mais, plus que l'empathie, je développe la thèse (et c'est ma contribution essentielle surce terrain, en lien avec la notion de PDV) de la mobilité empathique

9. Si l'empathie n'est pas la sympathie, elle s'en distingue mal dans les premiers âges des êtres humains. Ultérieurement, l'empathie, centralement cognitive, n'est pas en rupture totale avec les problématiques affectives et les phénomènes de consonance émotionnelle. Voir Tisseron, 2010 ; Brunel et Cosnier, 2012, etc. 
(Rabatel 2016a), c'est-à-dire l'idée que l'être humain gagne à changer de PDV. Cependant, avant d'aller plus loin, il convient de dire que l'empathie peut servir au pire (le stratège est empathique en prévoyant la tactique de l'adversaire) comme au meilleur, à la condition qu'elle soit mêlée de considération sympathique envers autrui (Nussbaum 2013, p.202-203). L'empathie est alors une chance pour le sujet psycho-social, qui, plutôt que de se replier sur soi, s'ouvre aux autres non seulement à ceux qui lui sont proches, mais aussi à ceux qui sont éloignés de lui (spatialement, sociologiquement, culturellement, idéologiquement) ; qui peut multiplier des expériences différentes tout en les hiérarchisant, pour essayer de penser le complexe. Dans cette optique, la démarche de mobilité empathique consiste non seulement à pratiquer cette mobilité en s'intéressant aux autres dans leur diversité, mais à la pratiquer aussi dans son rapport aux objets de l'expérience, en les envisageant sous plusieurs angles (Latour, 2006, p. 210-213), sous divers points de vue théoriques, pratiques, idéologiques.

La mobilité empathique favorise, outre le décentrement, un mode de compréhension de sujet à sujet ${ }^{10}$ : on empathise plus aisément sur des sujets individuels que sur les groupes ${ }^{11}$, qu'on risque d'essentialiser, de figer dans des stéréotypes (Rabatel, 2016b). D'où le lien entre l'empathie et un certain nombre de capabilités au fort potentiel démocratique (Nussbaum, 2011, p.37-38 et 61; Nussbaum, 2013, p.341-343), pour former des êtres solidaires, essayant de penser les intérêts de groupe, de se mettre à la place des logiques institutionnelles, de ne pas se cantonner dans une position basiste défiante envers «ceux qui sont en haut», pour forger du social et du commun, voire prendre conscience de solidarités avec d'autres formes de vie.

Donc : il n'y a pas un seul point de vue, mais une multiplicité de points de vue partiels, avec des degrés de pertinence et de vérité relatifs. Cependant, la dimension relative et historique des points de vue, loin de donner nécessairement lieu à une vision totalement relativiste du savoir qui a encore souvent cours - il n'y aurait que des PDV partiaux, limités, qui ne pourraient jamais donner lieu à une élaboration objective globale -, donne au contraire des arguments pour penser que, dès lors que les PDV sont situés, mobiles, inscrits dans le temps, ils peuvent évoluer, se discuter, se combiner, donner lieu à des vérifications, des expérimentations, et donc entrer dans une logique scientifique et objectivante qui est en mesure de fournir des vérités, certes toujours révocables à mesure de l'évolution, mais néanmoins acceptables par une communauté si rien ne les contredit (Rabatel 2013C).

10. Voir Morin, 1999, p. 52 ; Nussbaum, 2011, ch. 6 ; Nussbaum, 2013, p. 203-204; Nussbaum, 2015, p. 43-44 et 156-158.

11. Ce qui ne veut pas dire qu'il ne faut empathiser que sur les sujets; M. Nussbaum (2011, p. 37) a raison de souligner que, d'un point de vue politique, il est important de se mettre à la place de groupes, prendre en compte les différences et contradictions qui les traversent : cela vaut $e . g$. pour les instances de décision, les partis, les groupes socio-économiques envers lesquels il faut défendre ses intérêts (de personne ou de groupe) et négocier. 
Dans ce cadre très général, il y a place autant pour la raison raisonnante que pour les émotions, dont les raisons sont aussi des ressorts essentiels de la réflexion et de l'action. De même qu'il y a des communautés discursives, il y a des communautés émotionnelles, des gens avec qui on partage des émotions, ce qui nous rappelle que ces dernières ont aussi une dimension sociale structurante (Monte et al., 2015), irriguent les projets politiques, les idéologies, les esthétiques, qui sont autant des façons de penser son rapport au monde et au pouvoir que des façons de vivre et de ressentir. Les émotions (surtout les émotions secondaires), analysées dans une perspective empathique, peuvent déboucher sur des évolutions des modes de ressentir et de penser, surtout si elles sont considérées dans leurs dimensions psycho-sociales.

J'ai par exemple analysé comment la police ou la justice pourraient évoluer dans leur façon de traiter des plaintes des femmes dans les cas de viol, si, au lieu de se contenter d'opposer les témoignages des deux parties, qui souvent se neutralisent, elles cherchaient (éventuellement avec l'aide des experts, par exemple des spécialistes de traumatologie) à se mettre à la place des victimes, et à penser certaines incohérences des témoignages à l'aune d'un vécu traumatisant (Rabatel, 2015d). Ces déficits des institutions sont aussi partagés par les médias, dans l'affaire Nafissatou Diallo contre Dominique Strauss-Kahn comme dans l'affaire des suicides à France Télécom. Là aussi, les médias commencent par opposer les témoignages des syndicalistes à ceux de France Télécom en se bornant à reproduire les déclarations des directions locales, sans jamais interroger les dirigeants nationaux (alors que les suicides se multiplient partout sur le territoire national) et sans recueillir des avis d'experts ou des témoignages de collègues (Rabatel, 2010a). Il faut attendre une forte conjonction de suicides à l'été 2009, dont certains spectaculaires, pour que la presse se fasse l'écho de l'Observatoire sur les suicides, traite des suicides en les inscrivant dans une série, adopte des hyperstructures qui donnent la parole à des experts, des salariés, citent des chiffres, évoquent d'autres suicides, dans d'autres entreprises, pour que la question ne soit plus réduite à des fragilités individuelles mais renvoie à un problème social et politique d'organisation du travail. Les journalistes interrogent enfin les dirigeants nationaux des entreprises et ministres, puis proposent dans leur propre discours des analyses globales du phénomène (Rabatel 2011b, c). Dans les deux cas, il faut faire effort pour se mettre à la place des uns et des autres, faire la synthèse des témoignages, les jauger, les confronter à des sources autres, mettre en avant des responsabilités, et prendre ses propres responsabilités en investiguant. Or cela n'est pas facile, pas seulement parce qu'il est interdit de pénétrer dans les entreprises, de recueillir des témoignages de médecins du travail soumis au secret professionnel, de collègues de travail du suicidé qui ne veulent pas parler parce qu'ils se sentent mauvaise conscience, mais aussi parce qu'il fallait changer de mode d'écriture (donc pas seulement réécrire les 
dépêches d'agence), changer de mode de raisonnement, pour ne pas traiter les faits comme des cas isolés, personnels, mais comme des symptômes de dysfonctionnements sociaux... J'ajoute que l'analyse empathique est intéressante lorsqu'elle s'interroge sur le choix et la nature des décentrements cognitifs et/ou émotifs : il est par exemple significatif que dans l'expression de son repentir public sur TF1, D. Strauss-Kahn présente des excuses à sa femme, sa famille, au peuple français, pas à ses camarades et pas non plus à N. Diallo (Rabatel, 2016c), que les émotions concernent sa peur, son indignation, et non N. Diallo, qui n'est évoquée qu'à travers sa lecture du rapport du procureur.

MOTS. Les langages du politique : Vous vous êtes intéressé au fonctionnement des médias dans leur rapport avec le politique : quel rôle pourraient-ils jouer dans un fonctionnement plus démocratique de l'État?

Alain RABATEL : Mes travaux ne proposent pas de solutions politiques ni ne critiquent du point de vue surplombant de qui aurait « la » vérité. Mais ils éclairent certains dysfonctionnements, en mêlant toujours considérations sociales ou politiques et considérations méthodologiques, linguistiques : quand j'analyse le traitement médiatique des suicides, c'est non seulement pour relever des responsabilités patronales, mais aussi des responsabilités médiatiques. C'est pourquoi je plaide pour que les journalistes puissent revendiquer de meilleures conditions de travail, faire des enquêtes, vérifier, pour qu'ils s'appuient sur le travail des chercheurs, et des spécialistes (pas simplement des experts autoproclamés ou désignés), en sollicitant les formes d'expertise sociale les plus diverses. Le rôle des médiateurs comme des décisionnaires est d'éclairer le peuple en s'appuyant sur toutes sortes d'expertises, en imaginant des scénarios, avec leurs retombées positives et négatives, qui aident à la prise de décision, et non d'être l'enregistreur passif ou le sollicitateur voyeuriste des opinions toutes faites, qui nous tirent tous vers le bas. À chaque fois que j'entends des journalistes des chaines publiques, dans les journaux télévisés, demander aux téléspectateurs ce qu'ils pensent de telle ou chose, en les invitant à participer à un sondage qui se réduit à un binarisme affligeant, sans avoir d'abord fourni des éléments de connaissance qui aideraient à la réflexion et à la décision éventuelle, j'ai envie de casser le poste (et c'est hélas plusieurs fois par jour). La presse n'a pas besoin de faire le lit du Front national pour aider à la propagation de ses modes de pensée simplistes, il suffit de reproduire sans discontinuer des modes de raisonnement primaires et de laisser penser que des problèmes (qui ne sont jamais présentés dans leur complexité) n’appellent que quelques mesures immédiates et un peu de «courage »12. Par conséquent, la question de la mobilité empathique est aussi capitale pour la conception

12. Et quand des partis politiques « républicains» jouent sur ce même registre, abdiquant ce qui fait la grandeur du politique, le pire est à craindre. 
que je me fais du travail des professionnels des médias et aussi de l'analyste des médias : j'attends (quelles que soient leurs convictions) qu'ils se donnent et nous donnent des clés pour penser le complexe en entrant dans les raisons des uns et des autres, et en offrant ainsi une compréhension aussi honnête et compréhensive que possible des forces en présence ou des logiques des évènements. Et de même pour l'analyste. J'ai besoin d'avoir des outils pour comprendre. Et cela en n'étant pas prisonnier des choix personnels qu'il est légitime d'avoir par ailleurs (Rabatel, 2013c et d).

MOTS. Les langages du politique : Au vu des caractéristiques essentielles que vous dégagez, seriez-vous d'accord pour dire que votre travail s'inscrit dans le courant de la Critical Discourse Analysis?

Alain RABATEL : Difficile de donner une réponse tranchée. Il n'est pas faux de souligner une certaine parenté entre les travaux de Van Dijk, Fairclough, Wodak et les miens. Mais mes préoccupations théoriques débordent le cadre de l'analyse de discours, et même dans ce cadre, mes travaux sont à la croisée de plusieurs courants de l'analyse de discours (Rabatel, 2011a, p. 176). D'autre part, mes travaux concernant les discours médiatiques se réclament d'une dimension politique, engagée, certes, mais essentiellement critique, et je suis gêné quand je vois des travaux se réclamant de la Critical Discourse Analysis se servir de leurs corpus comme de prétextes qui leur permettent d'asséner des vérités politiques indiscutables (Rabatel, 2013a, p.47).

MOTS. Les langages du politique : Vous avez défendu à plusieurs reprises au long de cet entretien une éthique du discours, tout en soulignant la subjectivité inhérente à toute prise de parole, fût-elle apparemment marquée par l'effacement énonciatif. Comment conciliez-vous ces deux convictions? Quelle forme peut prendre une éthique discursive dégagée des illusions naïves de l'objectivité? Alain RABATEL : Les travaux sur les PDV en confrontation, la réflexion sur des logiques et des scénarios différents, peuvent aider à mieux comprendre le caractère complexe des organisations humaines, et, ce faisant, mieux éclairer les choix des citoyens. L'action politique comme la pratique journalistique et le monde médiatique sont en difficulté et en retard par rapport à ces nécessités épistémologiques de penser le complexe en ayant le souci de faire émerger du commun (Dardot et Laval, 2014; Rabatel, 2016d). Il faut solliciter les réactions des individus, leurs expériences, sans se contenter de les juxtaposer, et provoquer des situations qui permettent d'articuler les expériences de sujet à sujet avec des démarches globalisantes, objectivantes. Sur ce plan, les nouveaux médias et les nouvelles technologies facilitent le développement des liens interpersonnels (dans lesquels toutefois la tentation narcissique est forte), mais restent très en deçà face à la nécessité de faire société en élaborant du commun. Enfin, j'ajouterai pour finir une double conviction qui 
a des répercussions importantes au plan méthodologique : je suis convaincu qu'il faut avoir un ancrage disciplinaire fort (en l'occurrence dans les sciences du langage dans leur diversité, pas seulement en Analyse de discours) pour pouvoir pratiquer des approches pluridisciplinaires ${ }^{13}$, soit en s'ouvrant à des travaux de chercheurs d'autres disciplines (Florea et Rabatel, 2011; Rabatel, Monte et Soares Rodrigues, 2015) soit en s'ouvrant soi-même, en tant que chercheur, à des analyses provenant d'autres disciplines, ce que montre la diversité des références bibliographiques de mes travaux, qui pratiquent une certaine interdisciplinarité, à partir de mon cadre théorique énonciatif, en articulant les analyses linguistiques minutieuses, avec l'extérieur, le social.

Outre que cela oblige à une réflexion critique sur le relativisme, comme je l'ai dit plus haut, cela devrait inciter à s'interroger sur l'idée que les médias n'auraient à faire qu'avec des représentations. Cette interrogation concerne autant les professionnels des médias que les chercheurs qui analysent le travail (et les conditions de travail) des journalistes. Faudrait-il se borner à juxtaposer des représentations, en abdiquant l'ambition de formuler des analyses cohérentes, vérifiées par la marche du monde et l'état des savoirs, en considérant comme inaccessible ou indue, au regard d'une objectivité dont nous avons vu plus haut ce qu'il fallait en penser, l'ambition de hiérarchiser, discuter, et proposer des vérités, fussent-elles relatives? C'est pourquoi j'ai écrit à plusieurs reprises que je partageais les réserves de l'historien Éric Baratay (il n'est pas indifférent que cette critique émane d'un historien, confronté à l'analyse critique des «faits») envers le «tout représentations » qui a pu accompagner un certain linguistic turn ${ }^{14}$. Car la notion de représentation ${ }^{15}$ a un intérêt épistémologique fondamental, si elle interroge de façon critique des opinions et leurs motivations. Mais c'est là un préalable, qui n'implique pas que les représentations soient la fin du travail scientifique, faute de quoi, en effet, tout se vaut, si on ne confronte pas la pertinence des représentations avec la réalité extralinguistique - quand bien même on sait que la réalité est aussi façon-

13. Comme Mots. Les langages du politique ou Questions de communication le revendiquent à juste titre.

14. «Une telle position est devenue commune, presque une vulgate, dans les sciences humaines, et notamment en histoire avec le succès de l'analyse culturelle qui étudie les représentations à l'œuvre dans les discours, leur circulation et leur évolution [...] Il faut dépasser, je ne dis pas abandonner, le jeu postmoderniste de la déconstruction des postures et des discours, qui, laissé à lui-même, fait oublier la notion de réalité, alors qu'il est lui-même obligatoirement construit, daté et sujet... à déconstruction, et qu'il ne peut pas être une fin en soi. Dans le cas de l'histoire, il faut dépasser le linguistic turn américain et l'histoire culturelle qui s'en est inspirée, parce qu'ils ont souvent incité l'historien au commentaire des mots donnés, pour écrire les représentations ou pour préserver les sciences du piège des lectures humaines. Ce travail est évidemment obligatoire, mais le triomphe des lectures culturelles a transformé ce préalable indispensable en finalité indépassable; ce qui n'était pas le cas chez les fondateurs de l'histoire culturelle. » (Baratay, 2012, p. 54-55)

15. Représentation, re-présentation : ces notions et variations méritent d'être théorisées, leurs usages précisés, voir Florea et Rabatel, 2011. 
née par les discours - et on abdique toute prétention à influer, à sa place, sur le cours des choses.

Comme je n'abdique pas, je plaide pour une analyse engagée et responsable des discours médiatiques. Et, pour terminer sur une note positive, j’observe que, dans les nouvelles générations, ce souci trouve un écho qui va croissant. C'est pourquoi j'espère que le gros volume rassemblant l'essentiel de mes travaux sur les médias, qui devrait paraitre dans le courant de l'année (Rabatel 2017c), rencontrera un public en consonance avec les choix que je défends.

\section{Références bibliographiques ${ }^{16}$}

BARATAY Éric, 2012, Le point de vue animal, Paris, Éditions du Seuil.

Berthoz André, 2004, "Physiologie du changement de point de vue», L'empathie, A. Berthoz, G. Jorland dir., Paris, Odile Jacob, p. 253-275.

Brunel Marie-Lise, CoSNIER Jacques 2012, L'empathie. Un sixième sens, Lyon, Presses universitaires de Lyon.

DARDot Pierre, LAVAL Christian, 2014, Commun : essai sur la révolution au XXIe siècle, Paris, La Découverte.

Ducrot Oswald, 1984, Le dire et le dit, Paris, Éditions de Minuit.

FLOREA Marie-Laure, 2015, Les nécrologies dans la presse française contemporaine. Une analyse de discours, Thèse de doctorat : Sciences du langage, université Lumière Lyon 2 〈http://www.theses.fr/2015LYO20122/document〉 (consulté le 15 novembre 2016).

FLoREA Marie-Laure, RABATEl Alain, 2011, «Les modes de re-présentation de la mort et leurs enjeux dans la construction de l'événement», Questions de communication, n'20, p. 7-18.

KRIEg-PlAnque Alice, 2009, La notion de «formule» en analyse de discours. Cadre théorique et méthodologique, Besançon, PUFC.

LATOur Bruno, 2006, Changer de société, refaire de la sociologie, Paris, La Découverte. Monte Michèle, Rabatel Alain, SOARES Rodrigues Maria Graças, 2015, «La dynamique des émotions dans l'affaire Nafissatou Diallo versus Dominique Strauss-Kahn », A. Rabatel, M. Monte, M. G. Soares Rodrigues éd., Comment les médias parlent des émotions. L'affaire Nafissatou Diallo contre Dominique Strauss-Kahn, Limoges, Lambert-Lucas, p. 7-35.

MoRIN Edgar, 1999, Les sept savoirs nécessaires à l'éducation du futur, Paris, Unesco/ Éditions du Seuil; disponible au format PDF 〈http://unesdoc.unesco.org/ images/0011/001177/117740fo.pdf> (consulté le 15 novembre 2016).

Nussbaum Martha, 2011 [2010], Les émotions démocratiques. Comment former les citoyens du xxı siècle? Paris, Flammarion.

16. Bibliographie complète disponible sur internet 〈http://icar.cnrs.fr/membres/arabatel〉 (consulté le 3 février 2017). 
Nussbaum Martha, 2013 [2012], Les religions face à l'intolérance. Vaincre la politique de la peur. Paris, Climats.

Nussbaum Martha, 2015 [1995], L'art d'être juste. Paris, Flammarion.

Rabatel Alain, 1997, Une histoire du point de vue, Metz, Université de Metz, Centre d'études linguistiques des textes et des discours.

RABATEl Alain, 1998, La Construction textuelle du point de vue, Lausanne, Lausanne, Delachaux et Niestlé.

RABATEl Alain, 2004, "La déliaison des énonciateurs et des locuteurs dans la presse satirique», Langage et société, $\mathrm{n}^{0}{ }^{110}$, p. 7-23.

RABATEL Alain, 2005, "La part de l'énonciateur dans la construction interactionnelle des points de vue», Marges linguistiques, nº 9, p.115-136; disponible en ligne au format PDF 〈http://www.revue-texto.net/Parutions/Marges/oo_mlo92005.pdf〉 (consulté le 18 novembre 2016).

RABATEL Alain, 2006, "L'effacement de la figure de l'auteur dans la construction événementielle d'un “journal” de campagne électorale et la question de la responsabilité, en l'absence de récit primaire », Semen, n²2, p. 71-85.

RABATEl Alain, 2008a, "Pour une conception éthique des débats politiques dans les médias : répondre de, devant, pour, ou les défis de la responsabilité collective», Questions de communication, $\mathrm{n}^{0} 13$, p. 47-69.

RABATEL Alain, 2008b, Homo narrans. Pour une analyse énonciative et interactionnelle du récit. Tome 1. Les points de vue et la logique de la narration. Tome 2. Dialogisme et polyphonie dans le récit, Limoges, Lambert-Lucas, 2 vol. RABATEL Alain, 2009a, «Perspective et point de vue», Communications, n85, p. 23-35.

RABATEL Alain, 2009b, «Prise en charge et imputation, ou la prise en charge à responsabilité limitée », Langue française, $n^{\circ} 162$, p. 71-87.

RABATEL Alain, 2010a, «Deux modes de représentation idéologique people du pouvoir, lors du premier anniversaire de la présidence de N. Sarkozy », Semen, nº 30, p. 147167.

RABATEl Alain, 2010b, "Analyse pragma-énonciative des s/citations du site d'Arrêt sur images", Argumentation et analyse de discours, n० 4 <http://aad.revues.org/ index806.html> (consulté le 15 novembre 2016)

RABATEL Alain, 2010c, "Le traitement médiatique des suicides à France Télécom de mai-juin à mi-août 2009 : la lente émergence de la responsabilité du management dans les suicides en lien avec le travail », Studia Universitatis Babes-Bolyai, Philologia, t. LV, vol.1, p. 31-52.

RABATEL Alain, 2011a, "La levée progressive du tabou des responsabilités socio-professionnelles dans les suicides en lien avec le travail à France Télécom, de fin août à octobre 2009", Questions de communication, n²0, p. 175-198.

RABATEL Alain, 2011b, "Des conflits de valeurs et de points de vue en discours», Semen, $n^{\circ} 32$, p. 55-72.

RABATEL Alain, 2011c, «Figures d'à-peu-près et nom propre», Le français moderne, t. LXXIX, vol. 1, p. 22-33.

RABATEL Alain, 2012a, «Sujets modaux, instances de prise en charge et de validation », Le Discours et la langue, t. $3, \mathrm{n}^{\circ} 2, \mathrm{p} .13-36$. 
RABATEL Alain, 2012b, «Positions, positionnements et postures de l'énonciateur », Travaux neuchâtelois de linguistique, $\mathrm{n}^{\circ} 56$, p. $23-42$.

RABATEL Alain, 2013a, "Les apports de l'analyse des discours médiatiques : de l'interprétation des données à la critique des pratiques discursives et sociales », Dacoromania, vol.XVIII, nº1, p. 33-50.

RABATEL Alain, 2013b, «Empathie et émotions argumentées en discours », Le Discours et la langue, vol. 4, $\mathrm{n}^{\circ} 1$, p. 159-177.

RABATEL Alain, 2013c, «Éthique, point(s) de vue et rapport aux différents régimes de vérité», Le rapport éthique au discours, C. Guérin, G. Siouffi, S. Sorlin éd., Berne, Peter Lang, p. 65-80.

RABATEL Alain, 2013d, "L'engagement du chercheur, entre "éthique d'objectivité" et "éthique de subjectivité" ", Argumentation et analyse de discours, nº11 〈http:// aad.revues.org/1526> (consulté le 18 novembre 2016)

RABATEl Alain, 2014a, "La rubrique Intox/désintox de Libération. Nouvelle rubrique, nouvelle pratique journalistique, voire nouveau genre?», Des textes aux genres, Hommages à Jean-Michel Adam, M. Monte, G. Philippe, éd., Lyon, Presses universitaires de Lyon, p. 103-116.

RABATEL Alain, 2014b, “"La parole des politiques soumise à contre-enquête” représente-t-elle de la même façon la parole des hommes et des femmes politiques?", Discours rapporté genre(s) et médias, F. Sullet-Nylander, M. Roitman, J.-M. LopezMunoz, S. Marnette, L. Rosier dir., Stockholm, Stockholm University, Romanica Stockolmiensia, p.197-208.

Rabatel Alain, 2015a, "Retour sur un parcours en énonciation", Points de vue sur le point de vue. Un essai de réflexion collective, M. Carcassonne, A. Cunha Doris, C. Donahue, F. François, A. Rabatel, Limoges, Lambert-Lucas, p. 327-355.

RABATEL Alain, 2015b «Postures énonciatives, variable générique et stratégies de positionnement», in Analyse du discours et dispositifs d'énonciation. Autour des travaux de Dominique Maingueneau, J. Angermuller, G. Philippe dir., Limoges, Lambert-Lucas, p.125-136.

RABATEL Alain, 2015C, "Du style en politique dans les commentaires métadiscursifs médiatiques des deux premières années de présidence de N. Sarkozy», Style, langue, société, É. Bordas éd., Paris, Honoré Champion, p. 371-381.

Rabatel Alain, 2015d, "Dégoût et indignation dans le Manifeste/pétition féministe Pas de justice, pas de paix», in Comment les médias parlent des émotions. L'affaire Nafissatou Diallo contre Dominique Strauss-Kahn, A. Rabatel, M. Monte, M. G. Soares Rodrigues éd., Limoges, Lambert-Lucas, p.143-160.

RABATEL Alain, 2016a, «Diversité des points de vue et mobilité empathique », in L'énonciation aujourd'hui, un concept-clé des sciences du langage, M. Colas-Blaise, G.-M. Tore, éd., Limoges, Lambert-Lucas, p. 135-150.

RABATEl Alain, 2016b, "De la difficulté de catégoriser le peuple (des invisibles) en échappant aux jugements de valeur», Exercices de rhétorique, $\mathrm{n}^{0} 7$ 〈http://rhetorique.revues.org/457> (consulté le 15 novembre 2016).

RABATEL Alain, 2016c, «Les stratégies émotives d'un repentir public offensif », Studii de Lingvistică, nº6, p.109-125. 
RABATEL Alain, 2016d, «Analyse de discours et inégalités sociales : de l'empathie pour les invisibles à l'engagement pour le commun », Revista de estudos da linguagem, vol. $14, n^{\circ} 27$, p. $757-788$.

RABATEL Alain, 2017a (à paraître), «Éthique des discours, prise en charge et responsabilité énonciatives », in Éthique des discours et responsabilité, G. Yanoshevsky dir., Limoges, Lambert-Lucas.

RABATEL Alain, 2017b (à paraître), «Les parcours interprétatifs des présupposés et des sous-entendus, de la phrase au texte et à sa mise en page », La présupposition en discours, A. Biglari, M. Bonhomme éd., Paris, L'Harmattan.

RABATEL Alain, 2017c (à paraître), Pour une lecture linguistique et critique des médias, Limoges, Lambert-Lucas.

Rabatel Alain, Chauvin-Vileno Andrée, 2006, «La question de la responsabilité dans l'écriture de presse», Semen, n²2, p.5-24.

RABATEl Alain, Koren Roselyne, 2008, «La responsabilité collective dans la presse», Questions de communication, $\mathrm{n}^{0} 13, \mathrm{p} \cdot \mathrm{7}-18$.

Rabatel Alain, Monte Michèle, Soares Rodrigues Maria Graças éd., 2015, Comment les médias parlent des émotions. L'affaire Nafissatou Diallo contre Dominique Strauss-Kahn, Limoges, Lambert-Lucas.

Rizzolatti Giacomo, Sinigaglia Corrado, 2007, Les neurones miroirs, Paris, Odile Jacob.

TISSERon Serge, 2010, L'empathie au cœur du jeu social, Paris, Albin Michel. 